\title{
A Smart Campus Template
}

\author{
Juan Carlos AUGUSTO ${ }^{\mathrm{a}, 1}$ \\ ${ }^{a}$ Research Group on Development of Intelligent Environments \\ Department of Computer Science, Middlesex University London, UK
}

\begin{abstract}
We highlight a lack of models and theories associated with the Smart Campus concept and also an absence of processes to support its design and development. This paper provides a first approach to a theory and a set of design principles to guide their development. The theory and principles are flexible enough to be easily adapted and adopted by any organization interested in developing a Smart Campus.
\end{abstract}

Keywords. Smart Campus, Intelligent Environments, Human-centric Computing

\section{Introduction}

Ask yourself the question: which has been the latest worldwide adopted revolution in technology-mediated education? Mostly likely we will agree on: PowerPoint-style of digital slides. Later on "smart" whiteboards became a product, some educational organizations acquired them and in some countries they are fairly common, however they did not offer much in the way of smartness in the clever sense rather facilitated digitalization of content. This for "smart education" and "smart classrooms" $[1,2,3]$. When we escalate the look at "Smart Campus" level, the concept is mentioned on the web, however, it has been mostly hijacked by certain organizations which have done some new equipment acquisition, again, abusing a technical term which is meant to signify something more substantial. Uptake from Universities have been slow. Universities are busy exporting innovation outside their walls and often forget to incorporate technical innovation themselves.

Despite the occasional bad use of the term it is a worthy concept to adopt, we can apply it properly, and reap the rewards of innovating in this direction. It can use state of the art technology to introduce efficiencies for students, staff, and administration. Smart Campus can help connecting people and resources. It can be designed to address in a balanced and ethical way the preferences and needs from all main University stakeholders. It could be a positive stimulus both internally and externally for more innovation.

The following articles provide a picture of the state of the art at an international level. Janelle et al., [4] investigated the spatially enabled campus through a multi-disciplinary team including technological, institutional, and social perspectives. For the specific organization conducting that introspective assessment it triggered the collection of ideas and evidence to support future development in that direction and emphasized their internal priorities on: sustainability, knowledge sharing, cost effectiveness, student involvement and learning, safety, and other perspectives. Kwok [5] Highlights the role of data, procedural knowledge, and system integrations as resources as well as privacy concerns and the time it takes to develop the concept, as challenges. It emphasizes the importance of networks, computers, systems, processes, people and knowledge

\footnotetext{
${ }^{1}$ Corresponding Author: Juan Carlos Augusto; E-mail: j.augusto@mdx.ac.uk.
} 
extraction processes as part of the essential infrastructure required to materialize the concept. Sari et al. [6] provides an example of a smart campus design from Indonesia focused on the use of IoT for the development of smart campus services limited to education, parking and rooms automation. Bi et al. [7] provides an example from China focusing on the use of Building Information Modeling (BIM) and of 3D Geographic Information System (3DGIS). Chan and Chan [8] looks at the latest development of Smart Campus and, especially, Smart Libraries, focusing on how innovation in those areas create positive impact on those who use their services. Muhamad et al. [9] investigates the kind of technology used, the models used, the services created, and the perceived benefits. It highlights the growth in activity within this area of innovation and the diversification of services beyond teaching and learning, for example sustainability and management support. This article highlights the need for services to be reactive and dynamic, and highlights the challenges in the areas of interaction and interoperability. Min-Allah and Alrashed [10] distills a Smart Campus approach from Smart City ones: People into Community, Planet into Campus Infrastructure, Prosperity into Sustainability/Employability, Governance into Administration/Management, and Propagation into Replicability/Innovation. They notice the absence of a single vendor/provider of services, the absence of global standards and the possibility to attract investments through small scale projects with clear business returns to make the project self-sustainable.

Despite these isolated proposals and initial explorations there is still little consensus on the fundamental principles and underpinning theories, no methods and no tools, which are immediately helpful to develop this concept. This paper aims at providing a first unified conceptual view that build up on previous experiences and captures the essence of the main concepts at stake and hopefully act as a community discussion starter.

\section{Smart Campus as an Intelligent Environment}

Here we take the view that a Smart Campus is an instance of an Intelligent Environment [11], in this case one created to support those who make use and interact with the campus.

Definition: "The 'Smart' technology, associated software, and processes, which facilitate the main objectives of the campus."

Here 'smart' is understood as sensing/actuation technology which supports contextaware decision-making. This is related to the following areas in Computer Science: Ubiquitous Computing, Pervasive Computing, Intelligent Environments, Internet of Things, Ambient Intelligence, and their unifying principle: context-awareness [12].

Important components are sensors, actuators, and interfaces linked by networks, and supplemented by middleware which facilitate the context-awareness. These can also be complemented by AI software, mobile computing, robotics. etc.

There is no shortage of technological resources, for example: (passive) screens (relying information), 'smart' whiteboards, advanced interfaces (e.g., voice processing, image processing, haptics, etc.), sensing technology (e.g., to measure use of a resource), smart phones and their apps, data (storing/analysing/visualization), mixed reality, virtual presence, robots, artificial intelligence (e.g., real-time context-awareness, machine learning, etc.).

On one hand tools shape what we can do and how, however technology in an intelligent environment is only relevant to the extent it contributes to provide to the stakeholders the services they expect [13]. Determining what the stakeholders expect is 
the starting point, however these systems are so complex they require some iterations through suggesting services, mapping to infrastructure and checking these with stakeholders until they converge to a desirable, and also feasible, project (hence the bidirectional arrows in figure 2.1).

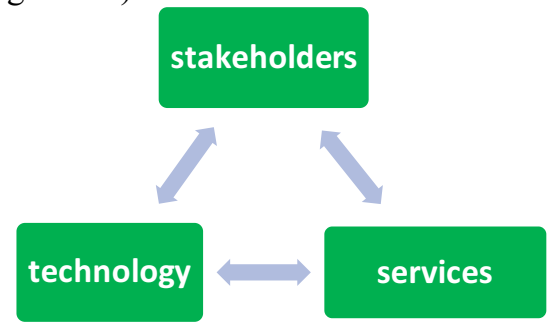

Figure 2.1 Fundamental Smart Campus Triad

We expand on the triad stakeholders-services-technology of important inter-related concepts in the subsections below, giving stakeholders more importance as they will greatly affect what services are created and which infrastructure is considered.

\subsection{Stakeholders}

We advocate here for a stakeholders-led system creation, such as the User-centred Intelligent Environments Development Process [14]. In terms of identifying stakeholders, obviously this can be conducted at all sort of granularity levels depending on how specific the services are. At an initial stage of a Smart Campus an obvious higher level partition of stakeholders can make emphasis on the main groups of activities and responsibilities, for example (figure 2.2):

- Teaching and Learning related: these can comprise students of taught courses and those delivering the material to be learnt,

- Research and Innovation related: these could be trainees such as PhD students and also supervisors and lab assistants, and

- Decision-making and Support-related: these comprise different groups of staff with the task of supporting the operational activities of the organization at all levels, this includes, for example, senior managers and technicians.

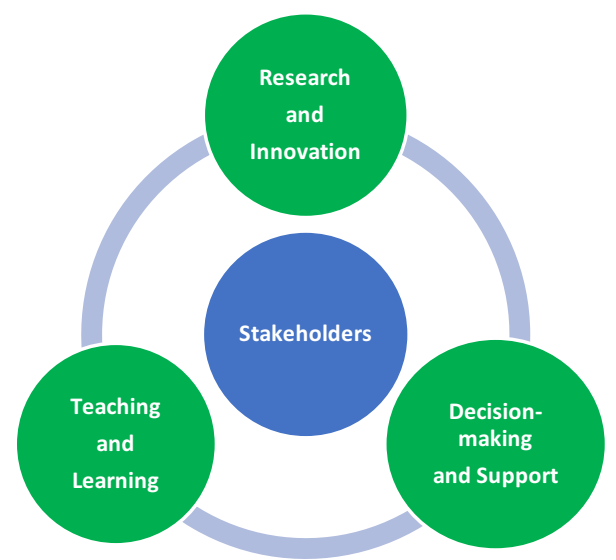

Figure 2.2. Core Smart Campus stakeholder groups 
We emphasize these should not be taken as the definitive stakeholder groups, only as an illustration of the concept and there are interesting subtleties to consider. On one hand these categories can be looked at a finer grain, for example, distinguishing amongst undergraduate and postgraduate students. Additional subtleties include that some stakeholders can be in more than one category, for example a member of staff can be pursuing further studies and being a stakeholder both as staff and as student, or a member of staff can have decision-making roles such as Director of Program or Research Degrees Coordinator which are complementary to the usual staff responsibilities. On the other hand stakeholders categories seem static however they are dynamic when looked at a granularity of months/years and people can move through stakeholder categories. For example, from student to alumni, from student to staff, from teaching staff to decisionmaker as Head of Department.

\subsection{Services}

Services are the specific benefits which stakeholders ultimately get from the system. Each University may be able to offer different services and also may have different internal priorities, so it is not possible to provide a prescription, however, some options associated with campus activity linked to the most generic stakeholder groups can be:

- To support learning activities: accessing information, facilitating learning and assessment, health and wellbeing of students, etc.

- To support innovation activities: creativity, training, coaching/mentoring, connecting and collaborating, etc.

- To support decision-making activities: connecting with students/staff, optimizing services, keeping services operational, support in identifying and reacting to critical events, etc.

We are using these three categories as a way to illustrate the overall concept without getting ourselves lost in too many details, however every University have to work out their stakeholders organization which best represent their situation and focus.

Echoing what we stated about stakeholders changing stakeholder group, services associated with those individuals may have to travel with the person to the next stakeholder category s/he is entering to. And of course services themselves may change with time as the organization changes priorities and resources availability.

\subsection{Technological infrastructure}

Ultimately the enabler of the Smart Campus concept will be the use that humans give to the selected technology. There are plenty of options now on technologies, examples of recent technologies which are tempting to consider in relation to various services are: (passive) Screens (relying information), "smart" whiteboards, advanced interfaces (e.g., voice/image processing, haptics), sensing technology (e.g., to measure use of a resource), smart phones and their 'apps', data (storing/analyzing/visualization), mixed reality, virtual presence, robots, artificial intelligence (e.g., real-time context-awareness, and machine learning).

In fact there are so many interesting gadgets and devices and people is heavily bombarded with news from companies about each of these that often projects end up being technology led. Many projects which are not carefully managed end up as a cocktail of gadgets and systems with low acceptance and short life. Following a more 
stakeholders centred approach and making technology the consequence of decisions instead of the cause should increase the chances to end up with a safer Smart Campus system.

\section{Landing the concept}

A Smart Campus, like any Smart Environment, is heavily based on its physical spaces. A campus typically has the following spaces: classrooms, lab rooms, offices, meeting rooms, corridors, food areas, socialization areas, toilets, walk ways, storage areas, sport areas, etc. Some of these areas are more often used by different groups of stakeholders, some areas are more specialized and attract users with more specific aims, others will provide services to all, for example a food area. Areas in the campus can be identified according to their priority intended use, services available and perhaps also which stakeholders can have access to them, which also has implications with security. For example, consider Figure 3.1 and assume the outer shape represents the campus, the inner six rectangles represent six different buildings and the smaller four rectangles inside represent rooms. Let us assume the services clusters are those related to: Teaching and Learning related activities (represented with "T"), Research and Innovation related activities (represented with "R") and Decision-making related activities (represented with "D"). This represents different rooms have services expected to cater for different focal areas of the organization. For simplicity we considered only three here, however, naturally there could be more areas with complementary functions associated, for example, areas for interaction and socialization such as 'common rooms', cafes, eating areas, faith related areas, and of course there are also areas which are meant to be used by everyone such as corridors and patios.

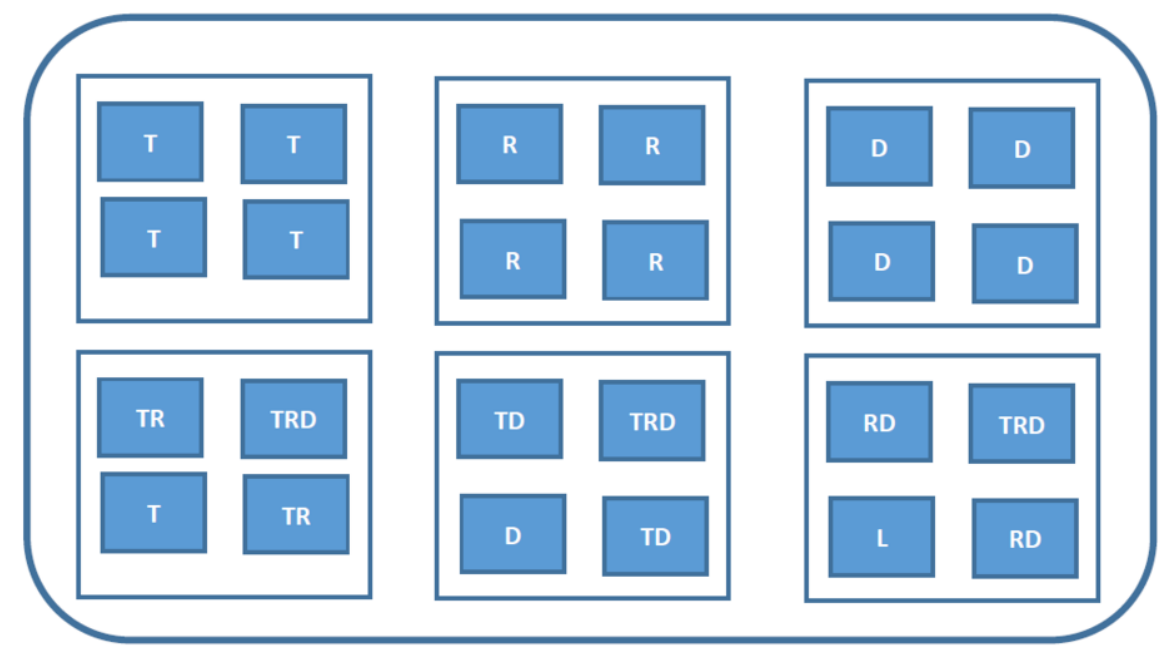

Figure 3.1. Combinations of "Smart Spaces"

Next we consider some scenarios which we use to illustrate how the concepts above can be interlinked. Again, they are not intended to be prescriptive, rather act as examples and motivators for future developers to adapt to their specific project. Figure 3.2 shows an extract of the previous figure where we highlight spaces dedicated to provide services of 
a specific type, as we clarified before in practice spaces can, and often will, combine services of different categories serving different groups of stakeholders.

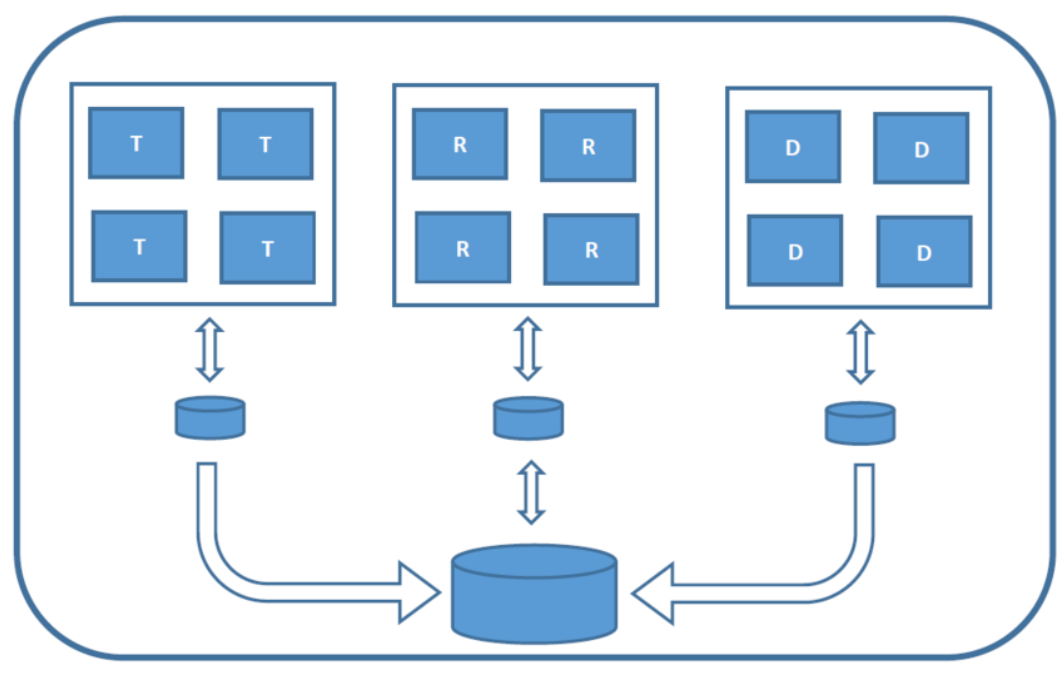

Figure 3.2. Services template for scenarios

\subsection{Scenario 1}

Main focus: to support stakeholders related to learning and teaching activities.

Services:

- Students are alerted through their an app the lecture starts in a certain room, they are warned today the lecture takes in a different place, the orientation app helps them find the place from where they are.

- Once in the classroom students attendance is confirmed.

- The lecturer starts the class the smart whiteboard help retrieving previous digital material, drawing and writing newly generated material, storing in digital form this new material. Content is multi-media (e.g., text, audio, video, drawings, documents, augmented reality files). Material can be now accessed in a multimedia channel.

- Side screens allows Distance Learning students to connect and participate.

- App to capture disruptive students (contextualized recording only operable by staff).

- When room idle is empty lights go off, when out of expected academic expected use, security can be alerted if noisy.

Possible Infrastructure to achieve them:

- Smart whiteboard;

- Extra screen to support Distance Learning;

- Recording and Storage of selected material; and

- Light management through movement sensors. 


\subsection{Scenario 2}

Main focus: to support stakeholders related to innovation activities.

Services:

- Support spontaneous collaboration or funding call driven.

- Apps to connect colleagues by technical interest ('theme pals' nearby).

- Library services to bring relevant information more easily.

- Multi-touch screens on mobile frames:

- To concentrate "creativity apps";

○ To support "out of the box" ideas;

- For formation; and

- Progression.

- Simultaneous collaboration; and

- Easy conversion of collaboration into easily transportable digital records of interaction.

Possible Infrastructure to achieve services:

- Flexible access to information and knowledge repositories; and

- Creativity stimulating environments.

\subsection{Scenario 3}

Main focus: to support stakeholders with higher decision-making responsibilities.

Services:

- It should provide:

○ centralized and distributed; and

○ synchronous and asynchronous communication.

- Issues tracking (challenges and progress highlighting).

- Meeting and interaction facilitation.

- Solutions support:

- Relevant Data facilitation; and

- Creative brainstorming facilitation.

- Planning support.

- "Firefighting" support.

Possible Infrastructure to achieve services:

- Data analytics;

- Data visualization; and

- Creativity support.

\section{A Reusable Template}

How this fit in the wider Computer Science landscape? We can see the concept of Smart Campus as an instantiation of the wider concepts of Ubiquitous Computing related systems, a family of areas which developed in the last three decades using sensing to produce systems which are useful to humans in practical daily life situations, especially here we explore more deeply the definition in section 2 at system level. 
Most distinctive to these systems is their input data coming from sensors, which complements that more traditional from interfaces and databases, updating in real-time the contexts relevant to the services the system is supposed to deliver. These "Smart" systems also use actuators to produce an effect in the physical environments where they operate. By "sensors" here we mean artifacts which can translate parameters from the physical world into digital information and by "actuators" we mean artifacts which can translate digital information into manifestations in the physical world. Smart systems by their very nature are created to benefit humans in specific environments and contexts [12] and are meant to consider the system stakeholders preferences and needs and optimize certain stakeholders satisfaction represented by a function $\Omega$ (Fig. 4.1).

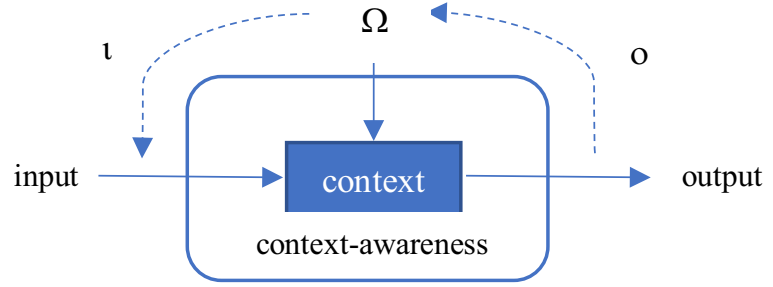

Fig 4.1 Context-aware system reactive to services optimization function $\Omega$

These systems, in whatever way have been implemented and regardless of how consciously their developers were based in these premises above always try to implement a system which provides some value to some stakeholders. Ideal full global satisfaction may be impossible as sometimes the preferences of some stakeholders conflict with those of other stakeholders. Some systems have well defined hierarchies and that defines how certain services are given priority over others. There may be a number of optimal and suboptimal levels of system performance.

Like all control systems, these systems can have dynamic feedback loops ("o", omicron, in Figure 4.1) informing updates on the optimization function $\Omega$ and adjusting the allowed inputs (" 1 ", iota, in Figure 4.1). Often environments have multiple groups of stakeholders with overlapping and also different service expectations and for a system to satisfy all stakeholders a global optimization function needs to represent these and be managed by the system. That is $\Omega$ can be defined as a function $\Omega\left(\omega_{1}, \ldots, \omega_{\mathrm{n}}, \mathrm{O}_{1}, \ldots, \mathrm{O}_{\mathrm{m}}\right)$ defined on a number of stakeholders groups $\omega_{1}, \ldots, \omega_{\mathrm{n}}$ and a number of Organizational Objectives $\mathrm{O}_{1}, \ldots, \mathrm{O}_{\mathrm{m}}$. Each $\omega_{\mathrm{i}}$ is the outcome of a function taking into account each stakeholder $\mathrm{s}_{\mathrm{j}}$ of that stakeholder group, collectively or as an addition of individual's functions and each organizational objective. Each $\mathrm{O}_{\mathrm{j}}$ is set by the organization (with feedback from other stakeholders), they can be revised periodically and provide the main comparison reference to understand how well the system is serving stakeholders. Examples of organizational objectives could be: to produce innovation, to train and graduate new professionals, and to have a positive impact in society, we leave them generically indicated in the examples that follow, we will come back to them at the end of this section.

In the reminder of this section we offer some indications on how these feedback loops work for the three scenarios outlined in the previous section. For example, in Scenario 1: "o" could channel the recent organization desire of augmenting health over other service clusters, now $\Omega\left(\omega_{\text {learners, }} \omega_{\text {teachers, }} \omega_{\text {decision-makers, }} \mathrm{O}_{1}, \ldots, \mathrm{O}_{\mathrm{m}}\right)$ will take in the results of each of these stakeholder polls resulting on an adjusted " $i$ " from what it was 
(let us say, "safety $>$ health $>$ pedagogy $>$ sustainability") into "health $=$ safety $>$ pedagogy $>$ sustainability" which is used then to affect the decisions from the contextawareness modules leading to for example, more messages in screens about health related issues around areas designated as having a role in teaching and learning ("T" type in figure 3.1). Another example, for Scenario 2: "o" reflects now the aim of the organization to augment inter-faculty scientific cooperation amongst colleagues so $\Omega\left(\omega_{\text {innovators}}, \omega_{\text {decision-makers, }} \mathrm{O}_{1}, \ldots, \mathrm{O}_{\mathrm{m}}\right)$ will tune in the system input accordingly through "l" from what it was (e.g., "information finding $>$ creativity $>$ cooperation $>$ sustainability") into "cooperation $>$ creativity $>$ information finding" and amongst the practical consequences of this adjustment the context- awareness modules will prioritize selecting funding calls which are labelled as multidisciplinary for circulation and encourage technical "meetups" amongst relevant colleagues. Again as a way of an example, for Scenario 3: "o" can reflect the adjusted priority from the higher strategic decision-makers of the University that the organization should have increased emphasis on knowledge transfer activities so $\Omega\left(\omega_{\text {students, }} \omega_{\text {innovators, }} \omega_{\text {decision-makers, }} \mathrm{O}_{1}, \ldots, \mathrm{O}_{\mathrm{m}}\right)$ will tune in the system input through " $\mathrm{l}$ " to encourage participation on such activities by placing aim related activities to be favoured "(Knowledge Transfer $>\ldots$ otherorganizational-aims ..." and amongst the practical consequences of this adjustment the context- awareness modules will prioritize the external dissemination of highlights on successful innovation and coaching events with academic and marketing staff.

Figure 4.2 shows a complementary view of the process, highlighting how different stakeholders provide feedback which is then used to measure how well the experience of the stakeholders is aligned with the overarching organizational objectives and this is used by the system to make decisions that can help improve the experience of stakeholders.

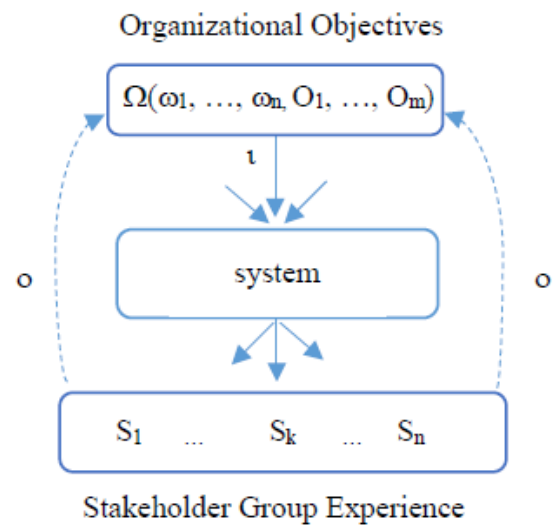

Fig 4.2 A wider view of the organizational feedback loop

One way to implement $\Omega$ is through a set of metrics $M$ in the system to measure indicators in "o" of how close is the stakeholders' experience $\omega_{\mathrm{i}}$ from what the organization would like them to have. Once a gap is identified a number of candidate actions A known to have a beneficial effect on $\omega_{\mathrm{i}}$ can be selected. The outcome " $\mathrm{"} \mathrm{should}$ have as a side effect in the decision-making model that actions A are favoured.

This system only tries to automate basic services however the proposed Smart Campus concept will still be driven by humans and will be for humans to benefit, they decide on the main goals, and the way to achieve them and when to change these. 


\section{Conclusions}

We reviewed the technical literature on Smart Campus, noticing there is an over emphasis on technology and a lack of models and recipes which other colleagues can adapt and use. Here we proposed a model centred on stakeholders as the guiding principle to select which services make sense and which technology, acceptable by stakeholders, can facilitate those services.

Even the technologies and services deployed so far are mostly: underused, isolated, and uncoordinated. Although Smart Campus can grow from simple services upwards, it is desirable they do that as part of a more holistic strategy which needs to have been defined by stakeholders (this includes everyone in the University at all levels). After that process the Smart Campus concept can incorporate existing services, help to create additional ones, and blend them together.

We offer also a higher level view of the Smart Campus concept as an instance of so called Smart/Intelligent Environments. This model provides a theory behind the Smart Campus concept as a system which can aim towards a global satisfaction level to the campus community with regards to certain objectives considered as higher priority by the interaction with stakeholders.

\section{References}

[1] Augusto J.C. Ambient Intelligence: Opportunities and Consequences of its Use in Smart Classrooms. Innovation in Teaching and Learning in Information and Computer Sciences (Italics), 2009; 8(2):53-63, Taylor and Francis.

[2] Currie E., Harvey P. H., Daryanani P., Augusto J. C., Arif R., Ali A. An investigation into the eficacy of avatar-based systems for student advice. EAI Endorsed Transactions on e-Learning, 2016; 16(11):e5.

[3] Yang, J., Pan, H., Zhou, W. et al. Evaluation of smart classroom from the perspective of infusing technology into pedagogy. Smart Learn. Environ. 2018; 5, 20.

[4] Janelle, D. G., Kuhn, W., Gould, M., Lovegreen, M. Advancing the Spatially Enabled Smart Campus, Final Report. UC Santa Barbara: Center for Spatial Studies. 2014.

[5] Kwok, L. A vision for the development of i-campus. Smart Learn. Environ. 2015; (2) 2.

[6] Sari M., Ciptadi P., Hardyanto R. Study of Smart Campus Development Using Internet of Things Technology. IOP Conference Series: Materials Science and Engineering, 2016; (190), IAES International Conference on Electrical Engineering, Computer Science and Informatics, Semarang, Indonesia. IOP Publishing Ltd.

[7] Bi T., Yang X., Ren M. The Design and Implementation of Smart Campus System. Journal of Computers $2017 ; 12(6), 527-533.4$

[8] Chan, H., Chan, L. (2018) Smart Library and Smart Campus. Journal of Service Science and Management, $11,543-564$.

[9] Muhamad, W., Kurniawan, N. B., Suhardi, S., Yazid, S. Smart campus features, technologies, and applications: A systematic literature review. In Proceedings of 2017 International Conference on Information Technology Systems and Innovation, (ICITSI). 2018; 384-391. Institute of EE Inc.

[10] Min-Allah N., Alrashed S. Smart campus_-A sketch. Sustainable Cities and Society. 2020; (59). Elsevier.

[11] Augusto J.C., Callaghan V., Kameas A., Cook D.J., Satoh I. Intelligent Environments: a manifesto. Human-centric Computing and Information Sciences, 2013; 3:12. Springer.

[12] Augusto J. C., Quinde M. J., Oguego C. L., Gimenez-Manuel J. G. Context-aware Systems Architecture (CaSA). To appear in Cybernetics and Systems, Taylor and Francis. 2020.

[13] Augusto J. C., Muñoz Ortega A. User Preferences in Intelligent Environments. Applied Artificial Intelligence, 2019; 33(12):1069-1091, Taylor and Francis.

[14] Augusto J.C. A User-Centric Software Development Process. In Proceedings The 10th International Conference on Intelligent Environments (IE'14), 2014; 252-255. Shanghai. IEEE Press. 